\title{
Circular RNAs: Novel biomarkers for cervical, ovarian and endometrial cancer (Review)
}

\author{
YUCHUAN SHI $^{1 *}$, RUNHUA HE $^{1 *}, \mathrm{YU} \mathrm{YANG}^{2}, \mathrm{YU} \mathrm{HE}^{1}, \mathrm{KANG} \mathrm{SHAO}^{1}, \mathrm{LEI} \mathrm{ZHAN}^{1,3}$ and BING WEI ${ }^{1}$ \\ ${ }^{1}$ Department of Gynaecology and Obstetrics, The Second Affiliated Hospital of Anhui Medical University; \\ ${ }^{2}$ Cardiology Department, The First Affiliated Hospital of Anhui Medical University, Hefei, Anhui 230601; \\ ${ }^{3}$ Department of Obstetrics and Gynecology, Reproductive Medicine Center, The First Affiliated Hospital of \\ Anhui Medical University, Hefei, Anhui 230022, P.R. China
}

Received March 3, 2020; Accepted August 24, 2020

DOI: $10.3892 /$ or.2020.7780

\begin{abstract}
Cervical, ovarian and endometrial cancer are the three most common types of malignant tumor and the leading causes of cancer-associated death in women. Tumor debulking surgery followed by platinum and paclitaxel chemotherapy is the current treatment regime of choice. However, as a result of late diagnosis and chemoresistance, the survival rates of patients with advanced gynecological cancers remains unsatisfactory. Circular RNAs (circRNAs) are stable noncoding RNAs that are present in a wide variety of tissue and cell types. With the enhancement of RNA sequencing methods, increasing numbers of circRNAs have been identified, and their functions are gradually being revealed. In recent years, circRNAs have received increasing attention for their regulatory roles in cervical, ovarian and endometrial cancer. The aim of the present review was to summarize the possible mechanisms of recently identified circRNAs; we hypothesize that a novel diagnostic and therapeutic biomarker may be identified to prolong the survival time of patients with gynecological malignancies.
\end{abstract}

\section{Contents}

1. Introduction

2. CircRNA biogenesis

3. CircRNA functions

Correspondence to: Dr Bing Wei or Dr Lei Zhan, Department of Gynaecology and Obstetrics, The Second Affiliated Hospital of Anhui Medical University, 678 Furong Road, Hefei, Anhui 230601, P.R. China

E-mail: 517275960@qq.com

E-mail: 499329901@qq.com

*Contributed equally

Key words: cervical cancer, ovarian cancer, endometrial cancer, circular RNA, biomarker
4. CircRNAs as potential biomarkers of gynecological tumors

5. Conclusions

\section{Introduction}

Circular RNAs (circRNAs) differ from other types of RNA due to their covalently closed-loop structures, and as such, have received increasing research interest since their discovery in 1976 (1). As members of the non-coding RNA family, circRNAs have been considered as secondary byproducts of linear mRNA splicing events (2). However, recent advancements in experimental and next-generation RNA sequencing methods have helped to identity and elucidate the functions of various novel circRNAs (3). CircRNAs have been revealed to influence a number of disease processes, including those involved in cardiovascular diseases, cancer and neurological disorders. Increasing research has confirmed the aberrant expression of numerous circRNAs in gynecological tumors, which influence the occurrence and development of these malignancies through multiple channels. This provides further evidence for the potential of circRNAs as biomarkers for the diagnosis, treatment and prognosis of gynecological cancers. The aim of the present review was to summarize our knowledge of the biogenesis and function of circRNAs, and to discuss the advantages and limitations of circRNA as biomarkers for cervical, ovarian and endometrial cancer, with the aim to identify novel biomarkers.

\section{CircRNA biogenesis}

As the name implies, circRNAs are closed circular structures with covalently linked $3^{\prime}$ and 5' ends, which distinguish them from other types of RNA (1). Most circRNAs are generated by pre-mRNA splicing, and their circular structures make them relatively stable and resistant to RNase digestion (4,5). Due to their various different sources, circRNAs can be categorized into three types: i) Exonic circRNAs (ecircRNAs), which are the product of back-spliced exons; ii) intronic circRNAs (ciRNAs), the product of intronic sequences; and iii) exon-intron circRNAs (EliciRNAs), the products of circularized exons with introns 
retained between them (3). These circRNAs are distributed differently within cells; ciRNAs and EliciRNAs are primarily present in the nucleus, while ecircRNAs are largely located in the cytoplasm (6). There are also three primary models for the formation of circRNA loops, namely lariat-driven, intron-pairing-driven and RNA-binding protein (RBP)-driven circularization (Fig. 1). These models can be further divided into two mechanisms; direct back-splicing and exon skipping (1). In the lariat-driven circularization model (exon skipping), a splice site 30 nucleotides upstream of an exon is ligated into a site 50 nucleotides downstream, leading to exon-skipping and the formation of an RNA lariat consisting of several exons and introns. The introns are then removed to generate ecircRNAs (4). Additionally, exons are circularized with introns that are retained between the exons, resulting in the generation of EliciRNAs (2). For the intron-pairing- and RBP-driven circularization models (direct back-splicing), reverse complementary sequences such as Alu repeats (located in the upstream and downstream introns), and certain trans-acting activator RNA-binding proteins binding to each of the flanking introns, bring the splice donor and acceptor sites close enough to form a loop (7-9). As for circular intron RNA biogenesis, the conserved motifs at both ends, including a 7-nt GU-rich element near the 5 splice site and an 11-nt C-rich element near the branch-point site, are joined together (10) to prevent introns from forming looped branches, and instead promote the formation of a loop structure. The 3 'end of the intron is then relocated to the branch point to produce a stable circular structure.

CircRNAs are widely present in various human cell types. Their expression is cell type-specific; cells with low proliferative capacity, such as cardiomyocytes, have higher expression levels compared with cells that proliferate more readily, such as those in the liver (7). In addition, the high level of circRNAs in some tissues is primarily the result of accumulation, which is likely due to the high stability of these molecules. Due to a lack of free ends, circRNAs are more stable and resistant to RNase R compared with linear RNAs (11). Owing to these aforementioned features, we hypothesized that circRNAs may serve as novel biomarkers for the diagnosis and treatment of essential hypertension (12), inflammatory bowel disease (13) and a variety of different cancer types (14). For example, in hepatocellular carcinoma, hsa_circ_0001649 is more sensitive and specific than the known biomarker alpha fetoprotein (15). Another circRNA, hsa_circ_025016, has proven to be a potential plasma biomarker for the prediction of postoperative atrial fibrillation (16).

\section{CircRNA functions}

MicroRNA (miRNA/miR) sponging. An increasing number of scholars are focusing on the roles of circRNAs in cancer, of which miRNA sponging is a major mechanism (Fig. 2A). CDR1as/ciRS-7 is a well-known circRNA that acts as an miRNA sponge to inhibit tumor growth in breast, colorectal, gastric and cervical cancer (17-20). Another highly investigated, typical competing endogenous RNA is circHIPK3, which is also involved in antioncogenic processes. By sponging miR-558, circHIPK3 suppresses the malignant properties of bladder cancer cells, including migration, invasiveness and angiogenesis (21). Similarly, another study indicated that circHIPK3 overexpression significantly suppressed the proliferation, migration and invasiveness of osteosarcoma cells in vitro (22). Furthermore, circHIPK 3 was found to promote the proliferation and progression of gallbladder and lung cancer cells, potentially by sponging miR-124 $(23,24)$. These findings suggest that the properties of circRNAs are a double-edged sword.

Protein interactions. Another regulatory function of circRNAs is achieved through their ability to interact with RNA-binding proteins (Fig. 2B), the results of which depend on the protein (and circRNA) in question. For example, p21 and cyclin-dependent kinase 2 strongly and specifically bind with circFoxo3 to form a circFoxo3-Cdk2-p21 complex, which inhibits transition from the $\mathrm{G}_{1}$ to the $\mathrm{S}$ phase of the cell cycle (25). Additionally, Du et al (26) revealed that circFoxo3 could bind p53 and mouse double minute 2 homolog to promote the ubiquitination and further degradation induced by p53. circMbl is able to bind the mannose-binding lectin (MBL); Ashwal-Fluss et al (27) revealed that the biosynthesis of circMbl was affected by the levels MBL via a feedback loop mechanism, and the process of MBL pre-mRNA translation into circMbl was also affected by high levels of MBL protein. Moreover, circMbl was found to bind MBL and attenuate its bioavailability. Another study identified a number of other circRNAs that bind multiple different proteins, serving regulatory roles in cell functioning (28).

Gene expression regulation. The majority of the circRNAs that regulate gene expression through sponge activity are localized in the cytoplasm (Fig. 2C). However, recent research has reported a small number of circRNAs that are localized in the nucleus, which can regulate gene expression at the transcriptional level. Li et al (29) found that circEIF3J and circPAIP2 are localized to the nucleus and promote the expression of parental genes, which involves the RNA polymerase II, U1 small nuclear RNA and several promoter regions. ciRNAs identified by Zhang et al (10) were also revealed to act as positive regulators of RNA polymerase-II transcription, and to promote parental gene expression. Similarly, ci-ankrd52 and ci-sirt7 were revealed to interact with RNA polymerase II to modulate the transcriptional rate of parental genes by accumulating at the active transcription site (10). Moreover, FECR1 circRNA, which binds to the promoter region and recruits TET1 DNA demethylase, was revealed to regulate the expression of the FLI1 gene, inducing DNA demethylation (30). These aforementioned circRNAs include those of intronic, exonic and exon-intron origin.

Protein translation. Although circRNAs are members of the non-coding RNA family, increasing data has revealed that circRNAs are also involved in protein translation (Fig. 2D). Unlike linear RNAs, circRNAs lack 7-methylguanosine cap structures and poly(A) tails, which prevents ribosomal recognition and translation into protein. Since the discovery of the internal ribosome entry site, the translation of circRNAs has become evident (31). A recent study indicated that circ-ZNF609, which regulates myoblast proliferation, contains an open reading frame that can be translated into protein with in cap-independent manner (32). Circ-FBXW7 is abundantly expressed in the normal human brain, and encodes a novel protein that regulates the proliferation and cycling of cancer 


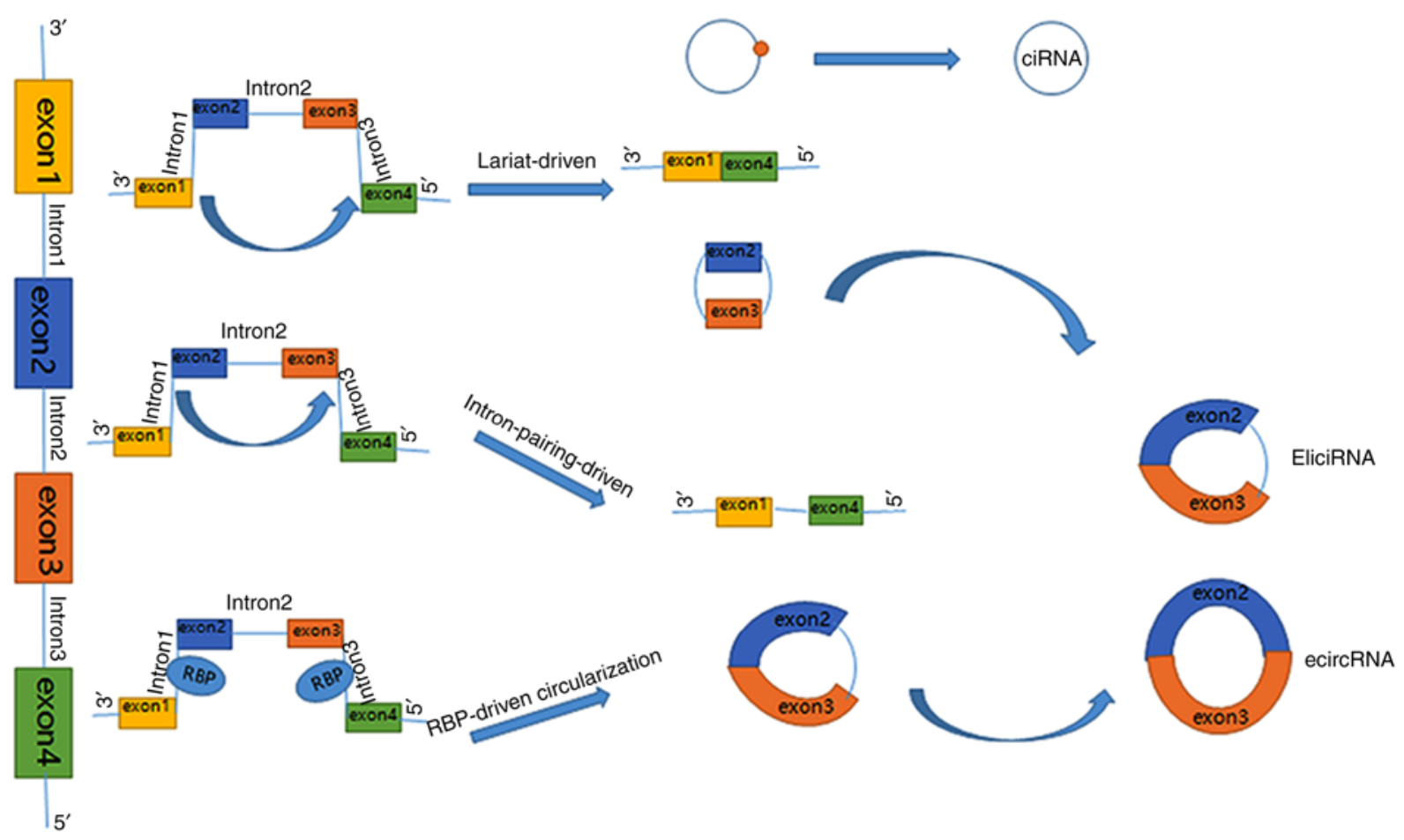

Figure 1. Biogenesis of circRNAs. CircRNA biogenesis is primarily regulated via three different mechanisms: Lariat-driven, intron-pairing-driven and RBP-driven circularization. circRNA, circular RNA; RBP, RNA-binding protein.

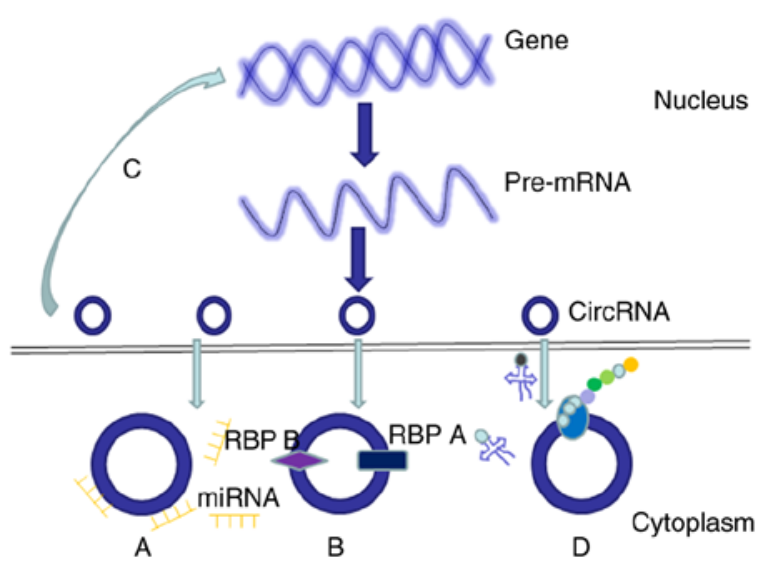

Figure 2. Functions of circRNAs. (A) CircRNAs act as miRNA sponges, regulating cellular proliferation. (B) CircRNAs bind to RBPs to regulate cell functions. (C) CircRNAs regulate the transcription of parental genes. (D) CircRNAs with IRESs can be translated into peptides or proteins. CircRNA, circular RNA; RBP, RNA-binding protein; IRES, internal ribosome entry site.

cells (33). Furthermore, circ-SHPRH encodes SHPRH-146aa, which regulates the proliferation and tumorigenicity of cancer cells in vitro (34). However, to date, few circRNAs have been found to be translated into protein, thus further studies in this area are warranted.

\section{CircRNAs as potential biomarkers of gynecological tumors}

A growing number of studies have focused on the relationship between gynecological tumors and circRNAs. The present review summarizes the most recent findings surrounding circRNA regulation in cervical, ovarian and endometrial cancer, with the aim to identify novel biomarkers and therapeutic approaches.

CircRNAs and cervical cancer (Table I). Cervical cancer is one of the most common malignant tumors in women, with an incidence rate second only to that of breast cancer (35). It has a high mortality rate and is the second major cause of tumor-related death worldwide (36). Continuous infection with high-risk human papillomavirus (HPV) is a major pathogenic factor for cervical cancer progression (37). Although the administration of HPV vaccines among patients with cervical cancer is increasing globally, mortality and morbidity rates remain high in numerous developed countries (38). In 2018, 570,000 newly-diagnosed cases of cervical cancer, and $\sim 311,000$ deaths, were recorded worldwide (39). Surgery followed by radiotherapy and chemotherapy is currently the primary method of cervical cancer treatment. However, the poor prognosis and low survival rate caused by distant metastasis and high lymphatic metastasis are still problematic (40). Therefore, the identification of novel treatments and biomarkers for earlier diagnosis is necessary for increasing survival rates.

A previous study revealed that circRNAs are stable and expressed to a high degree in various cancer cell lines (41). Additionally, circRNA detection methods are more sensitive and specific than those for the detection of proteins, and circRNAs are more easily detectable than miRNAs (42). For these reasons, there is a high probability that circRNAs will make suitable novel biomarkers for cancers. Currently, several studies have investigated the roles of circRNAs in cervical cancer, of which miRNA sponging may be the most significant 
Table I. Expression and functional characterization of circRNAs in cervical cancers.

\begin{tabular}{|c|c|c|c|c|c|}
\hline circRNAs & Expression & Binding miRNA & Function & Biomarker & (Refs.) \\
\hline Circ_0067934 & Up & $\operatorname{miR}-545$ & $\begin{array}{l}\text { Promotes cervical cancer } \\
\text { progression }\end{array}$ & Metastasis(+) & (48) \\
\hline circ-ATP8A2 & $\mathrm{Up}$ & miR-433 & $\begin{array}{l}\text { Promotes cell progression } \\
\text { and suppressing the expression } \\
\text { of epidermal growth factor } \\
\text { receptor }\end{array}$ & $\begin{array}{l}\text { Lymph node invasion and } \\
\text { FIGO stage }(+)\end{array}$ & (49) \\
\hline circ-0000745 & Up & NA & Acts as a tumor promoter & $\begin{array}{l}\text { Vascular/lymphatic } \\
\text { invasion }(+)\end{array}$ & (45) \\
\hline circ_0005576 & Up & miR-153-3p & $\begin{array}{l}\text { Promotes cervical cancer } \\
\text { progression }\end{array}$ & $\begin{array}{l}\text { Pathological stage and lymph } \\
\text { node metastasis(+) }\end{array}$ & $(50)$ \\
\hline circRNA_101308 & Down & $\begin{array}{l}\text { miR-26a-5p, } \\
\text { miR-196a-5p, } \\
\text { miR-196b-5p, } \\
\text { miR-335-3p, and } \\
\text { miR-1307-3p }\end{array}$ & Tumor suppressor & $\begin{array}{l}\text { Deep myometrial invasion } \\
\text { and lymph node metastasis(-) }\end{array}$ & (51) \\
\hline circSLC26A4 & Up & miR-1287-5p & Facilitates cancer progression & Poor prognosis & $(52)$ \\
\hline Circ_0018289 & $\mathrm{Up}$ & $\operatorname{miR}-497$ & Promotes tumorigenesis & Poor prognosis & (53) \\
\hline hsa_circ_0023404 & Up & $\operatorname{miR}-136$ & Promotes tumorigenesis & Poor prognosis & (54) \\
\hline circ-ITCH & Down & $\operatorname{miR}-93-5 p$ & $\begin{array}{l}\text { Suppresses proliferation and } \\
\text { metastasis }\end{array}$ & NA & (61) \\
\hline hsa_circ_0000263 & Up & miR-150-5p & NA & NA & (62) \\
\hline
\end{tabular}

circRNAs, circular RNAs; Ref., reference; NA, not available.

mechanism. The existing mechanisms (to the best of our knowledge) are discussed in detail below.

Expression of circRNAs in cervical cancer. Various studies have highlighted the presence of multiple circRNAs in cervical cancer. Among 526 dysregulated circRNAs, Zheng et al (43) found that 352 were upregulated and 174 were downregulated, indicating that circRNAs may be potential biomarkers for the diagnosis and treatment of cervical cancer. Gao et al (44) conducted a high-throughput microarray study using four pairs of cervical cancer and adjacent noncancerous tissues, revealing 19 downregulated and 26 upregulated circRNAs in cervical cancer. Jiao et al (45) studied circRNA expression profiles using microarray analysis, and found that 178 circRNAs were differentially expressed between cervical cancer and matched normal cervical tissues, of which 101 were downregulated and 77 were upregulated. By classifying the 77 upregulated circRNAs, they also identified 68 exonic, 3 intronic, 1 antisense and 5 intragenic circRNAs in cervical cancer tissues, as well as 101 downregulated circRNAs (including 81 exonic, 13 intronic, 3 antisense and 4 intragenic variants).

Although numerous studies aim to investigate the expression of ring-shaped RNAs in cervical cancer, there are often certain limitations. For example, the sample size of these studies is small, making it especially difficult to understand the expression profiles of different histological grades and pathological stages. It is therefore necessary to perform larger-scale sequencing studies to obtain more detailed data on the expression of circRNAs in cervical cancer.

i) CircRNAs as biomarkers of cervical cancer. The early diagnosis and treatment of cervical cancer can effectively improve the survival rates of patients. With the development of high-throughput sequencing and biochip technologies, a growing number of circRNAs have exhibited potential as diagnostic biomarkers.

ii) CircRNA expression in cervical cancer tissues samples. Research by Wang et al (46) highlighted that the expression level of hsa_circ_0001038 was higher in cervical cancer tissues than in adjacent non-cancerous tissues. Moreover, Chen et al (47) discovered that circRNA_0000285 was frequently upregulated in cervical cancer tissues compared with adjacent normal tissues.

iii) CircRNAs as biomarkers of cervical cancer metastasis. The expression of circ_0067934 was found to be increased in cervical cancer tissues and cell lines, compared with that in adjacent normal tissues (48). High circ_0067934 expression was also associated with positive lymphatic metastasis in patients with cervical cancer, indicating its use as a potential biomarker of cervical cancer metastasis. Ding et al (49) demonstrated that circ-ATP8A2 was upregulated in cervical cancer tissues and cell lines, and further confirmed that high levels of circ-ATP8A2 were associated with a higher FIGO 
stage, positive lymph node invasion and myometrial invasion. In addition, has-circ-u0000745 was upregulated in cervical cancer tissues and cell lines, which was found to be correlated with various clinicopathological features, such as poorly differentiated tumors and positive vascular/lymphatic invasion (45). Another circRNA, circ_0005576, has been associated with a higher pathological stage and lymph node metastasis (50). However, alternative studies have indicated that reduced circRNA expression also promotes metastasis; Jiao et al (51) revealed that low expression levels of circRNA_101308 were associated with deep myometrial invasion and lymph node metastasis. Therefore, selecting the appropriate circRNA biomarker for a specific pathology requires more thorough consideration.

CircRNAs as biomarkers of cervical cancer prognosis. It is important to identify patients with a poor prognosis in order to implement effective treatment. Therefore, there is an urgent requirement for the discovery of novel prognostic biomarkers for cervical cancer. CircRNAs are differentially expressed in tumors of different histological grades and pathological stages, and those with lymph node or distant metastases, suggesting that circRNAs have potential prognostic value as biomarkers of cervical cancer. For instance, Ji et al (52) revealed that the expression level of circSLC26A4 was upregulated in cervical cancer tissues compared with adjacent normal tissues, and that high circSLC26A4 expression was associated with a poor patient prognosis. Circ_0018289 has also been revealed to be markedly upregulated in cervical cancer tissues compared with adjacent normal tissues (53), which was significantly correlated with decreased overall survival, suggesting that circ_0018289 may be a potential biomarker for evaluating prognosis. Additionally, hsa_circ_0023404 was found to be upregulated in cervical cancer tissues and cell lines (54). As a result of Kaplan-Meier analysis, patients with high hsa_circ_0023404 expression levels exhibited poorer overall survival rates than those with low hsa_circ_0023404 expression levels. The primary associated mechanism was that hsa_circ_0023404 sponged miR-136, inducing yes-associated protein (YAP) pathway activation by promoting $\alpha$ globin transcription factor CP2 (TFCP2) expression, resulting in the development and progression of cervical cancer. This study revealed that hsa_circ_0023404 plays a pivotal role in the regulation of cervical cancer progression through the hsa_circ_0023404-miR-136-TFCP2-YAP axis. A number of other circRNAs, such as hsa_circ_0000515 (55), circ_0005576 (50) and circ-0000745 (45) have also been revealed to be highly expressed in cervical cancer, and associated with poor patient prognosis. The discovery of these circRNAs provides predictive possibility for the prognosis of cervical cancer.

CircRNAs suppress tumor progression. Cervical cancer results from the complex interaction between various factors and signaling pathways. Currently, the primary consensus is that this involves persistent infection with high-risk HPV. Multiple circRNAs have been revealed to play important roles in the progression of cervical cancer, and may therefore help to improve our understanding of the mechanisms underlying cancer progression.
CircSMARCA5 (hsa_circ_0001445), which is located on chr4:144464661-144465125 and forms the circular exon 15 and 16 of SMARCA5, is widely expressed in human cells. Previous studies have found that circSMARCA5 can regulate cellular proliferation by sponging miRNAs. For example, in prostate cancer, circSMARCA5 promoted tumor progression by sponging miR-432, but suppressed the progression of multiple myeloma by targeting miR-767-5p $(56,57)$. In cervical cancer, circSMARCA5 acted as a tumor suppressor with reduced expression in cancerous tissues, binding and inhibiting the expression of miR-620. High expression levels of circSMARCA5 suppressed the proliferation, migration and invasiveness of cancer cells by sponging miR-620 (58). CircRNA_101308 expression was also revealed to be decreased in cervical cancer tissues and cell lines, compared with that in normal tissues. CircRNA_101308 can bind various different miRNAs and regulate their downstream genes to suppress the proliferation, invasiveness and metastasis of cervical cancer cells, both in vitro and in vivo (51). Furthermore, circ-ITCH has been revealed to be associated with the regulation of tumor growth, and the proliferation and apoptosis of cancer cells in bladder (59) and breast cancer (60). In cervical cancer, circ-ITCH expression was low and functioned as an miRNA sponge for miR-93-5p. As the target of miR-93-5p, forkhead box protein $\mathrm{K} 2$ (FOXK2) regulated multiple cancer cell features. In cervical cancer, the overexpression of circ-ITCH suppressed the proliferation and metastasis of cervical cancer cells by interacting with the circ-ITCH/miR-93-5p/FOXK2 axis, and thus upregulating the expression of FOXK2 (61).

CircRNAs promote tumor progression. Previous research on circRNAs has improved our understanding of cervical cancer pathogenesis. Ma et al (50) recently discovered a novel circ_0005576-miR-153-3p-kinesin family member 20A (KIF20A) axis that regulates cervical cancer growth and invasion. They identified a potentially new form of circ_0005576 that is primarily located in the cytoplasm, and is significantly upregulated in cervical cancer. Circ_0005576-knockdown was found to suppress cellular proliferation, colony formation and metastasis, and circ_0005576 overexpression increased the expression of KIF20A by sponging miR-153-3p, which may provide a new perspective into the pathogenesis of cervical cancer. Moreover, hsa_circ_0000263 was revealed to be upregulated in cervical cancer cells, and cellular proliferation, migration and the cell cycle were inhibited by knocking down hsa_circ_0000263.In a murine model, the expression of murine double minute 4 (MDM4) was regulated by hsa_circ_0000263, which ultimately affected the expression of the p53 gene. Therefore, the hsa_circ_0000263/miR-150-5p/MDM4/p53 network has been suggested to serve a vital role in the regulation of cervical cancer (62). Furthermore, Hu et al (48) revealed that circ_0067934 was upregulated and associated with advanced stage, lymph node metastasis, and poor prognosis, providing new insights into the pathogenesis of cervical cancer. Tumor growth and cellular proliferation were inhibited by knocking down circ_0067934 in vitro and in vivo, and loss-of-function analysis revealed that increased miR-545 expression suppressed eukaryotic translation initiation factor 3 subunit C (EIF3C) expression by silencing circ_0067934. This study revealed that circ_0067934 influences cellular 
proliferation, migration and invasiveness in cervical cancer via the circ_0067934/miR-545/EIF3C axis.

CircRNAs as potential therapeutic targets. CircRNAs may represent potential targets for the treatment of cervical cancer. A previous study revealed that hsa_circ_0000515 was upregulated in cervical cancer, and acted as a ceRNA of miR-326 to increase the expression of ETS transcription factor (ELK1). The in vitro results indicated that proliferation and invasiveness, as well as induced apoptosis and autophagy, were suppressed by silencing hsa_circ_0000515 or overexpressing miR-326. ELK1 overexpression also suppressed apoptosis and autophagy, but enhanced the proliferation and invasiveness of cervical cancer cells. These findings indicate that hsa_circ_0000515 may be a tumor promoter and provide evidence for its therapeutic use in cervical cancer (55). Another circRNA, has-circ-0000745, was revealed to be upregulated in patients with cervical cancer, and high expression levels were associated with poorly differentiated tumors or vascular/lymphatic invasion. Knocking down hsa_circ_0000745 was found to upregulate E-cadherin expression, inhibiting the proliferation, migration and invasiveness of cervical cancer cells, indicating a potential target for cervical cancer treatment (45). Ding et al (49) revealed that circ-ATP8A2, which is highly expressed in cervical cancer specimens and cell lines, enhanced cellular proliferation, migration, invasiveness and apoptosis in cervical cancer. The same effects were observed for ectopically expressed circ-ATP8A2. The experimental results indicated that circ-ATP8A2 exerted its inhibitory effects on epidermal growth factor receptor (EGFR) expression at the post-transcriptional level, by sponging miR-433. These findings suggest a therapeutic effect of regulating the circ-ATP8A2/miR-433/EGFR axis to mediate tumor progression. Furthermore, the expression levels of circRNA_0000285 were higher in cervical cancer samples than in adjacent, non-cancerous tissues, and circRNA_0000285-knockdown in vitro significantly inhibited the proliferation and migration abilities of cervical cancer cells. circRNA_0000285 expression may also be correlated with that of the RNA-binding protein FUS, which was found to be regulated by circRNA_0000285. In a nude mouse model, the formation and metastasis of cervical cancer were significantly inhibited by circRNA_0000285-knockdown (47). Collectively, these data provide insights into the pathogenic mechanisms and potential treatment targets for cervical cancer.

Perspectives. At present, the association between cervical cancer and circRNAs is receiving extensive research attention. Researchers have identified numerous circRNAs that are aberrantly expressed in cervical cancer, as well as the molecular mechanisms underlying these phenomena. However, the mechanisms of a large number of circRNAs are yet to be elucidated. Currently, cervical cancer and normal adjacent tissues are the preferred specimen types for the study of circRNAs, and there is a lack of more readily available clinical specimen types, such as serum and urine, which would be more convenient for cervical cancer diagnosis. Therefore, additional research is required to improve our understanding of the relationship between circRNAs and cervical cancer.

CircRNAs and ovarian cancer (Table II). Ovarian cancer is a common, but life-threatening gynecological malignancy that represents $3.6 \%$ of neoplasms in women worldwide (63). Although it ranks 20th among the most common global cancer types, ovarian cancer has the highest mortality rate of all gynecological malignancies, and is the eighth leading cause of death among women worldwide $(63,64)$. In 2018, ovarian cancer resulted in an estimated 295,414 new cases and 184,799 deaths worldwide (39). Tumor debulking surgery followed by platinum and paclitaxel chemotherapy is currently the preferred clinical treatment (65). However, the survival rate of patients with advanced ovarian cancer is $\sim 30 \%$, which is primarily due to late diagnosis and chemoresistance, the latter of which is influenced by the tumor microenvironment and the inherent resistance of ovarian cancer cells to chemotherapy (66). Thus, improving the response to treatment and developing novel therapies is crucial for improving survival rates.

Expression of circRNA in ovarian cancer. Among 4,505 newly identified circRNAs, Teng et al (67) highlighted 2,431 that were significantly upregulated and 3,120 that were downregulated. Furthermore, circHIPK3 was indicated to regulate the proliferation and apoptosis of ovarian cancer and normal ovarian epithelial cells via the circHIPK3-miRNA-mRNA axis. Another study revealed that among 4,388 circRNAs with an expression fold change of $\geq 2,2,556$ were upregulated and 1,832 were downregulated. Further analysis indicated that differentially expressed circRNAs may serve a pathogenetic role in epithelial ovarian cancer, as well as acting as potential diagnostic and prognostic biomarkers (68). Moreover, of 710 differentially expressed circRNAs screened via high-throughput sequencing, circRNA1656 exhibited the highest fold change, with low expression in ovarian cancer tissues and cell lines, which was correlated with FIGO stage in high-grade serous ovarian cancer (69).

CircRNAs as biomarkers for the diagnosis, metastasis and prognosis of ovarian cancer. Early diagnosis prior to cancer metastasis significantly improves prognosis, and the prognostic evaluation of ovarian cancer also extends patient survival time (70). The pathogenesis of ovarian cancer is complex, and current diagnostic methods, including the detection of tumor markers, ultrasound, CT, MRI and histopathology, are not sufficient. Therefore, novel indicators for diagnosis, metastasis and prognosis are urgently required.

Researchers are now paying close attention to the regulation of circRNAs in ovarian cancer, with the aim to identify novel diagnosis biomarkers. Pei et al (71) discovered that hsa_circ_0013958 was highly expressed in ovarian cancer tissues and cell lines, and that hsa_circ_0013958 upregulation was closely associated with patient FIGO stage and lymph node metastasis. Further analysis revealed that hsa_circ_0013958 was a highly sensitive and specific indicator for the diagnosis of ovarian cancer. Previous studies have revealed that circLARP4 sponges miR-424 to regulate gastric cancer progression. In ovarian cancer, the expression of circLARP4 was found to be significantly downregulated, which was associated with FIGO stage and lymph node metastasis. Survival analysis revealed that a low level of circLARP4 was an independent risk factor for ovarian cancer prognosis, suggesting its potential use as a prognostic biomarker (72). Furthermore, circRNA_MYLK was revealed to be highly expressed in ovarian cancer compared 
Table II. Expression and functional characterization of circRNAs in ovarian cancers.

\begin{tabular}{|c|c|c|c|c|c|}
\hline circRNAs & Expression & Binding miRNA & Function & Biomarker & (Refs.) \\
\hline circMAN1A2 & Up & NA & NA & Serum biomarker & $(70)$ \\
\hline hsa_circ_0013958 & Up & NA & $\begin{array}{l}\text { Plays a role as an oncogene } \\
\text { in ovarian cancer }\end{array}$ & $\begin{array}{l}\text { FIGO stage and lymph } \\
\text { node metastasis }\end{array}$ & (71) \\
\hline circLARP4 & Down & $\operatorname{miR}-424$ & NA & Prognostic biomarker & $(72)$ \\
\hline CircRNA_MYLK & Up & microRNA-652 & $\begin{array}{l}\text { Promotes the malignant } \\
\text { progression }\end{array}$ & Prognostic biomarker & (73) \\
\hline hsa_circ_0051240 & Up & $\operatorname{miR}-637$ & $\begin{array}{l}\text { Promotes ovarian cancer } \\
\text { cell proliferation, migration } \\
\text { and invasion }\end{array}$ & NA & (74) \\
\hline circ-ABCB 10 & NA & $\begin{array}{l}\text { miR-1271 miR-1252 } \\
\text { miR-203 }\end{array}$ & $\begin{array}{l}\text { Promotes cell proliferation } \\
\text { but reduces cell apoptosis }\end{array}$ & $\begin{array}{l}\text { Poor prognosis and } \\
\text { advanced FIGO stage }\end{array}$ & $(75)$ \\
\hline Circ-SMAD7 & Up & NA & Promotes the progression & NA & $(76)$ \\
\hline VPS13C-has-circ-001567 & Up & NA & $\begin{array}{l}\text { Inhibits apoptosis and } \\
\text { promotes proliferation }\end{array}$ & NA & (77) \\
\hline hsa_circ_0078607 & Down & miR-518a-5p & Leads to cell apoptosis & NA & (79) \\
\hline circ_100395 & Down & $\operatorname{miR}-1228$ & $\begin{array}{l}\text { Inhibits cell growth and } \\
\text { metastasis }\end{array}$ & NA & $(80)$ \\
\hline circSETDB1 & Up & NA & $\begin{array}{l}\text { Predicts chemotherapy } \\
\text { response }\end{array}$ & Chemoresistant & $(81)$ \\
\hline CDR1as & Down & $\operatorname{miR}-1270$ & $\begin{array}{l}\text { Suppression of cisplatin } \\
\text { resistance, cell proliferation, } \\
\text { and apoptosis. } \\
\text { Enhances the sensitivity of } \\
\text { ovarian cancer to platinum }\end{array}$ & NA & $(82)$ \\
\hline
\end{tabular}

circRNAs, circular RNAs; Ref., reference; NA, not available.

with adjacent normal tissues, and high circRNA_MYLK expression was positively associated with pathological stage. Kaplan-Meier survival analysis indicated that high expression levels of circRNA_MYLK were closely related to a poor prognosis in patients with ovarian cancer (73). CiRS-7 has also been revealed to be upregulated in ovarian cancer, which is positively associated with TNM stage, lymph node metastasis status and poor prognosis. Moreover, Fan et al (70) discovered that circMAN1A2 was highly expressed in the serum of patients with various malignant tumors, including ovarian cancer, indicating its potential use as a serum biomarker. However, further clinical investigation is required to support these findings.

CircRNAs in tumor progression. Currently, the most common clinical treatment for ovarian cancer is tumor debulking surgery followed by platinum and paclitaxel chemotherapy. However, the poor prognosis of patients with advanced ovarian cancer remains a problem worldwide, and exploratory studies of pathogenesis and potential therapeutic methods are necessary. Zhang et al (74) revealed that hsa_circ_0051240 was upregulated in ovarian cancer compared with normal tissues, and that silencing hsa_circ_0051240 inhibited the proliferation, migration, invasion and tumor formation of ovarian cancer in vivo. The data also indicated that hsa_circ_0051240 was able to sponge miR-637, which directly targets KLK4
mRNA in ovarian cancer cells. It was therefore speculated that the hsa_circ_0051240-miR-637-KLK4 axis may be a potential treatment target for regulating the proliferation, migration and invasion of ovarian cancer.

circ-ABCB10 was also revealed to be upregulated in ovarian cancer, and was closely associated with large tumor size, poor differentiation, poor prognosis and an advanced FIGO stage. Chen et al (75) found that upregulated circ-ABCB10 expression reduced apoptosis but promoted cellular proliferation in vitro, and was also associated with the negative regulation of miR-1271, miR-1252 and miR-203. In addition, the upregulation of circ-SMAD7 negatively regulated Krüppel-like factor 6, mediating ovarian cancer progression, metastasis and cellular proliferation (76). Moreover, the capacity of VPS13C-has-circ-001567 to regulate the apoptosis and proliferation of ovarian cancer cells indicates that it may hold potential as a therapeutic target. VPS13C-has-circ-001567-knockdown inhibited the proliferation and tumorigenicity, but promoted the apoptosis of ovarian cancer in vitro; the cell cycle was arrested at the $\mathrm{G}_{1}$ phase, and the percentage of $S_{1}$ phase cells was decreased (77). Collectively, these findings suggest that circRNAs play indispensable roles in the regulation of ovarian cancer cell proliferation, migration and invasiveness, highlighting their potential use as therapeutic agents. 
CircRNAs suppress ovarian cancer progression. Chen et al (78) observed that circRNA CDR1as acted as a vital factor in the growth and metastasis of ovarian cancer. Hypoxia-inducible factor $1 \alpha$ inhibitor (HIF1AN) is an asparagine hydroxylase that is closely associated with cancer progression. As an miRNA sponge, CDR1 as inhibited miR-135b-5p to increase the expression of downstream HIF1AN, thus ultimately contributing to the suppression of ovarian cancer. hsa_circ_0078607 has also been found to be significantly downregulated in ovarian cancer (79). Additionally, hsa_circ_0078607 sponging of miR-518a-5p enhanced the expression of tumor necrosis factor receptor superfamily member 6 (Fas), which interacts with its receptor Fas ligand to induce the death signal cascade, resulting in apoptosis. This indicated that hsa_circ_0078607 suppressed ovarian cancer progression via the miR-518a-5p/Fas signaling pathway. Furthermore, Li et al (80) observed that circ_100395 facilitated the expression of p53 by modulating miR-1228, further inhibiting the growth and metastasis of ovarian tumors.

CircRNAs as biomarkers of ovarian cancer chemoresistance. Chemoresistance is a common phenomenon during cancer therapy that is caused by drug resistance, and results in treatment failure and poor patient prognosis. Chemoresistance frequently occurs in those with ovarian cancer, making it difficult to meet the chemotherapeutic standards of efficacy. It is therefore necessary to improve our understanding of the molecular mechanisms involved in ovarian cancer chemoresistance.

Wang et al (81) determined the levels of serum circSETDB1 in 60 patients with serous ovarian cancer, in order to evaluate its association with progression-free survival. They observed that higher levels of serum circSETDB1 were significantly associated with lymph node metastasis and advanced clinical stage, and could act as distinguishing indicators of patients and healthy volunteers. Notably, the levels of serum circSETDB1 were significantly increased in patients with primary chemoresistance, suggesting that serum circSETDB1 may be a predictor of progression, response to chemotherapy and relapse in ovarian cancer. To investigate the potential mechanisms involved, Zhao et al (82) evaluated the expression of circRNAs in cisplatin-resistant and -sensitive ovarian cancer tissues. Cisplatin-resistant patient tissues and cells exhibited lower expression levels of Cdrlas. Also, Cdrlas was determined to sponge miR-1270 and regulate the suppression of cancer cell invasiveness. These data indicate that ovarian cancer sensitivity to platinum may be enhanced by Cdrlas via the miR-1270/SCAI signaling pathway.

Perspectives. To date, the abnormal expression of numerous circRNAs has been detected in ovarian cancer, as well as the underlying mechanisms of some of these circRNAs. However, the resulting data are still limited by insufficient sample size and the lack of an expression profile for pathological classification. Therefore, further studies are required to fully address this clinical issue.

CircRNAs and endometrial cancer (Table III). Endometrial cancer is the fourth most common female malignant tumor worldwide, with $\sim 300,000$ newly diagnosed cases annually (39). The incidence of endometrial cancer is region-dependent, but is increasing with the aging of the population (83). Obesity, diabetes, polycystic ovary syndrome, infertility, early menarche and late menopause have all been reported as potential risk factors $(84,85)$. Although obesity and hyperinsulinemia in young women have increased significantly, the primary age of onset for endometrial cancer is in postmenopausal women, and the average age of cancer diagnosis is 63 years $(86,87)$. According to histological subtype, endometrial cancer can be classified into four types, including endometrioid, serous, clear cell and mixed endometrial cancer. It can also be divided into two types depending on the tumor response to estrogen. Type I tumors are estrogen-responsive, account for $\sim 80-90 \%$ of endometrial cancer cases, and are associated with a more favorable prognosis. By contrast, type II tumors are usually estrogen-independent, and are associated with a high grade and poor prognosis; despite accounting for only $10-20 \%$ of all cases, type II tumors are responsible for $40 \%$ of the total deaths from endometrial cancer (87-89). A recent study of overall mutational burden introduced novel classification criteria with different clinical outcomes. The Cancer Genome Atlas includes four types of endometrial cancer, including those with p53, polymerase epsilon and phosphatase and TENsin homolog mutations, microsatellite instability and histology (90). Early detection tends to result in improved prognosis; although postmenopausal bleeding is the most common symptom of endometrial cancer, it is a diagnostic indicator in only 5-10\% of cases. At present, the primary screening methods for endometrial cancer are transvaginal ultrasound scanning, outpatient hysteroscopy and endometrial biopsy. These procedures are expensive, and due to interference, can be difficult to perform effectively. Surgery combined with chemotherapy is currently the most commonly used therapeutic method for endometrial cancer, and a combination of carboplatin and paclitaxel is the first-line treatment of choice (91). However, poor prognosis with a rapid onset of resistance is still a significant issue. In summary, it is necessary to identity novel diagnostic and therapeutic methods to increase the survival rate of patients with endometrial cancer.

Expression and biomarker potential of circRNAs in endometrial cancer. A number of potential cancer biomarkers have been identified using high-throughput technologies. These include circRNAs, which serve regulatory roles in various physiological and pathological processes. In endometrial cancer, the roles of circRNAs in cells and tissues are gradually being revealed. Recent studies have shown that 75,928 unique circRNAs are abnormally expressed in endometrial cancer, and that 62,167 of these are significantly up- or downregulated in endometrial cancer tissues compared with adjacent normal tissues (92)C. The top 10 up- and downregulated circRNAs were selected for further investigation. Among them, the expression levels of hsa_circ_0001610 and hsa_circ_0039569 were lower in grade 1-2, compared with grade 3 endometrial cancer tissues, and age, tumor size, lymph node metastasis, myometrial invasion and FIGO stage were not associated with hsa_circ_0039569 expression level. However, hsa_circ_0001610 and hsa_ circ_0039569 were significantly associated with tumor differentiation. The correlation between these circRNAs 
Table III. Expression and functional characterization of circRNAs in endometrial cancers.

\begin{tabular}{|c|c|c|c|c|c|}
\hline circRNAs & Expression & Binding miRNA & Function & Biomarker & (Refs.) \\
\hline hsa_circ_0039569 & Up & miR-542 & NA & Diagnosis & (92) \\
\hline Circ-ITCH & NA & miRNA-17 miRNA-224 & NA & Diagnosis & (95) \\
\hline circWHSC1 & Up & miR-646 & $\begin{array}{l}\text { Promotes endometrial cancer } \\
\text { development }\end{array}$ & $\begin{array}{l}\text { Prognostic } \\
\text { biomarker }\end{array}$ & (97) \\
\hline
\end{tabular}

circRNAs, circular RNAs; Ref., reference; NA, not available.

and miRNAs was also analyzed, indicating that that the hsa_circ_0039569-has-miR-542-3p/has-let-7c-5p axis serves a regulatory role in grade 3 endometrial cancer. In summary, this study indicated that circRNAs may play a vital role in the diagnosis and treatment of grade 3 endometrial cancer, for which hsa_circ_0039569 may be a predictive indicator. Another study identified 209 upregulated and 66 downregulated circRNAs in extracellular vesicles, that were separated from the serum of patients with endometrial cancer. Of these 275 differentially expressed circRNAs, only hsa_circ_0109046 and hsa_circ_0002577 reached a fold-change $>2$, thus the potential interactions between these two circRNAs and miRNAs were predicted.

circRNAs have also been revealed to be potential biomarkers for the diagnosis of endometrial cancer (93). Chen et al (94) highlighted that the overall abundance of circRNAs is higher in the normal endometrium than in endometrial cancer tissues. Further research revealed 120 circRNAs that are differentially expressed between normal endometrial and endometrial cancer tissues, the majority of which are derived from exons. The transcriptional products of these circRNAs predispose endometrial tissues to malignancy. In summary, the results of the aforementioned studies indicate that circRNAs may serve as potential biomarkers for the diagnosis and progression of endometrial cancer.

The tumor suppressor gene PTEN plays an indispensable role in a number of biological processes, including cellular survival and apoptosis, proliferation and the maintenance of genomic stability. Circ-ITCH can combine with miRNA-17 and miRNA-224 to induce the differential expression of p21 and PTEN. In animal models, PTEN-knockdown promoted the development of endometrial cancer and its precursors (95). Also, women carrying germline PTEN mutations are at a higher risk of developing endometrial cancer (96), suggesting that different molecular mechanisms are involved in the regulation of circRNAs in this malignancy. Another study revealed that two circRNAs, HSPG2 and RP11255H23.4, are expressed in normal endometrial tissues, but not in endometrial cancer tissues. In normal tissues, the expression levels of the corresponding miRNAs were also increased, indicating that circRNAs can competitively bind to related miRNAs, promoting the development of endometrial cancer. The interaction between HSPG2 and various growth factors was also found to regulate endothelial growth and regeneration. Collectively, these findings suggest that circRNAs may act as diagnostic biomarkers and therapeutic targets for endometrial cancer.
CircRNAs in endometrial cancer progression. At present, few studies have investigated the roles of circRNAs in endometrial cancer progression. Liu et al (97) discovered that circWHSC1, which is highly expressed in cancer tissues compared with normal tissues, could regulate nucleophosmin 1 (NPM1), a downstream target of miR-646. This was subsequently found to be associated with the clinical stage and histological grade of endometrial cancer. CircWHSC1 may therefore promote the development of endometrial cancer by sponging miR-646 and targeting NPM1.

\section{Conclusions}

Bioinformatics and sequencing techniques have been used to identity numerous novel circRNAs involved in the development of cancer. Current studies indicate that circRNAs play an important role in the regulation of cellular proliferation, migration and invasiveness in cervical, ovarian and endometrial cancer, primarily via the miRNA sponging mechanism. Due to the stability of circRNAs, they possess great potential in tumor diagnosis and treatment. Furthermore, circRNAs can regulate gynecological tumors through a variety of molecular mechanisms, and blocking these pathways may represent novel therapeutic methodologies.

Although sequencing techniques have identified a growing number of circRNAs, their functions in gynecological cancer are still largely unclear. Moreover, the sample populations of recent studies have been relatively small and from a single study center, thus the reliability of the results cannot be fully guaranteed. Therefore, the introduction of more clinically available sample types, such as serum and urine, would improve study convenience. In addition, current research is primarily focused on the sponging function of circRNAs, with limited research into other functions. Moreover, due to the complexity of tumor pathogenesis, the mechanisms of circRNAs in tumors are not particularly clear, and further research is required to investigate these mechanisms in more depth.

Based on current research, the present review summarizes the potential of circRNAs as novel biomarkers for the diagnosis and treatment of cervical, ovarian cancer and endometrial cancer. However, further research is required to support these findings.

\section{Acknowledgements}

Not applicable. 


\section{Funding}

This project was supported by the National Natural Science Foundation of China (grant no. 81802586).

\section{Availability of data and materials}

Not applicable.

\section{Authors' contributions}

YS and RH contributed to the conception of the manuscript and wrote the draft. YY collected and prepared the literature. YH and KS revised the manuscript. LZ and BW provided funding and proofread the manuscript. All authors read and reviewed the final manuscript version.

\section{Ethics approval and consent to participate}

Not applicable.

\section{Patient consent for publication}

Not applicable.

\section{Competing interests}

The authors declare that they have no competing interests.

\section{References}

1. Sanger HL, Klotz G, Riesner D, Gross HJ and Kleinschmidt AK: Viroids are single-stranded covalently closed circular RNA molecules existing as highly base-paired rod-like structures. Proc Natl Acad Sci USA 73: 3852-3856, 1976.

2. Chen LL: The biogenesis and emerging roles of circular RNAs. Nat Rev Mol Cell Biol 17: 205-211, 2016.

3. Han B, Chao J and Yao H: Circular RNA and its mechanisms in disease: From the bench to the clinic. Pharmacol Ther 187: 31-44, 2018.

4. Jeck WR, Sorrentino JA, Wang K, Slevin MK, Burd CE, Liu J, Marzluff WF and Sharpless NE: Circular RNAs are abundant, conserved, and associated with ALU repeats. RNA 19: 141-157, 2013.

5. Enuka Y, Lauriola M, Feldman ME, Sas-Chen A, Ulitsky I and Yarden Y: Circular RNAs are long-lived and display only minimal early alterations in response to a growth factor. Nucleic Acids Res 44: 1370-1383, 2016.

6. Jeck WR and Sharpless NE: Detecting and characterizing circular RNAs. Nat Biotechnol 32: 453-461, 2014.

7. Bachmayr-Heyda A, Reiner AT, Auer K, Sukhbaatar N, Aust S, Bachleitner-Hofmann T, Mesteri I, Grunt TW, Zeillinger R and Pils D: Correlation of circular RNA abundance with proliferation-exemplified with colorectal and ovarian cancer, idiopathic lung fibrosis, and normal human tissues. Sci Rep 5: 8057, 2015.

8. Kramer MC, Liang D, Tatomer DC, Gold B, March ZM, Cherry S and Wilusz JE: Combinatorial control of drosophila circular RNA expression by intronic repeats, hnRNPs, and SR proteins. Genes Dev 29: 2168-2182, 2015.

9. Conn SJ, Pillman KA, Toubia J, Conn VM, Salmanidis M, Phillips CA, Roslan S, Schreiber AW, Gregory PA and Goodall GJ: The RNA binding protein quaking regulates formation of circRNAs. Cell 160: 1125-1134, 2015.

10. Zhang Y, Zhang XO, Chen T, Xiang JF, Yin QF, Xing YH, Zhu S, Yang L and Chen LL: Circular intronic long noncoding RNAs. Mol Cell 51: 792-806, 2013.

11. Suzuki H, Zuo Y, Wang J, Zhang MQ, Malhotra A and Mayeda A: Characterization of RNase R-digested cellular RNA source that consists of lariat and circular RNAs from pre-mRNA splicing. Nucleic Acids Res 34: e63, 2006.
12. Zheng S, Gu T, Bao X, Sun J, Zhao J, Zhang T and Zhang L: Circular RNA hsa_circ_0014243 may serve as a diagnostic biomarker for essential hypertension. Exp Ther Med 17: 1728-1736, 2019.

13. Ye YL, Yin J, Hu T, Zhang LP, Wu LY and Pang Z: Increased circulating circular RNA_103516 is a novel biomarker for inflammatory bowel disease in adult patients. World J Gastroenterol 25: 6273-6288, 2019.

14. Lei B, Tian Z, Fan W and Ni B: Circular RNA: A novel biomarker and therapeutic target for human cancers. Int J Med Sci 16: 292-301, 2019.

15. Meng S, Zhou H, Feng Z, Xu Z, Tang Y, Li P and Wu M: CircRNA: Functions and properties of a novel potential biomarker for cancer. Mol Cancer 16: 94, 2017.

16. Aufiero S, Reckman YJ, Pinto YM and Creemers EE: Circular RNAs open a new chapter in cardiovascular biology. Nat Rev Cardiol 16: 503-514, 2019.

17. Kong D, Piao YS, Yamashita S, Oshima H, Oguma K, Fushida S, Fujimura T, Minamoto T, Seno H, Yamada Y, et al: Inflammation-induced repression of tumor suppressor miR-7 in gastric tumor cells. Oncogene 31: 3949-3960, 2012.

18. Liu S, Zhang P, Chen Z, Liu M, Li X and Tang H: MicroRNA-7 downregulates XIAP expression to suppress cell growth and promote apoptosis in cervical cancer cells. FEBS Lett 587: 2247-2253, 2013.

19. Zhang H, Cai K, Wang J, Wang X, Cheng K, Shi F, Jiang L, Zhang Y and Dou J: MiR-7, inhibited indirectly by lincRNA HOTAIR, directly inhibits SETDB1 and reverses the EMT of breast cancer stem cells by downregulating the STAT3 pathway. Stem Cells 32: 2858-2868, 2014.

20. Zhang N, Li X, Wu CW, Dong Y, Cai M, Mok MT, Wang H, Chen J, Ng SS, Chen M, et al: microRNA-7 is a novel inhibitor of YY1 contributing to colorectal tumorigenesis. Oncogene 32: 5078-5088, 2013.

21. Li Y, Zheng F, Xiao X, Xie F, Tao D, Huang C, Liu D, Wang M, Wang L, Zeng F and Jiang G: CircHIPK3 sponges miR-558 to suppress heparanase expression in bladder cancer cells. EMBO Rep 18: 1646-1659, 2017.

22. Xiao-Long M, Kun-Peng Z and Chun-Lin Z: Circular RNA circ_HIPK3 is down-regulated and suppresses cell proliferation, migration and invasion in osteosarcoma. J Cancer 9: 1856-1862, 2018.

23. Yu H, Chen Y and Jiang P: Circular RNA HIPK3 exerts oncogenic properties through suppression of miR-124 in lung cancer. Biochem Biophys Res Commun 506: 455-462, 2018.

24. Kai D, Yannian L, Yitian C, Dinghao G, Xin Z and Wu J: Circular RNA HIPK3 promotes gallbladder cancer cell growth by sponging microRNA-124. Biochem Biophys Res Commun 503: 863-869, 2018.

25. Du WW, Yang W, Liu E, Yang Z, Dhaliwal P and Yang BB: Foxo3 circular RNA retards cell cycle progression via forming ternary complexes with p21 and CDK2. Nucleic Acids Res 44: 2846-2858, 2016.

26. Du WW, Fang L, Yang W, Wu N, Awan FM, Yang Z and Yang BB: Induction of tumor apoptosis through a circular RNA enhancing Foxo3 activity. Cell Death Differ 24: 357-370, 2017.

27. Ashwal-Fluss R, Meyer M, Pamudurti NR, Ivanov A, Bartok O, Hanan M, Evantal N, Memczak S, Rajewsky N and Kadener S: circRNA biogenesis competes with pre-mRNA splicing. Molecular Cell 56: 55-66, 2014.

28. Yang Q, Du WW, Wu N, Yang W, Awan FM, Fang L, Ma J, Li X, Zeng Y, Yang Z, et al: A circular RNA promotes tumorigenesis by inducing c-myc nuclear translocation. Cell Death Differ 24: 1609-1620, 2017.

29. Li Z, Huang C, Bao C, Chen L, Lin M, Wang X, Zhong G, Yu B, Hu W, Dai L, et al: Exon-intron circular RNAs regulate transcription in the nucleus. Nat Struct Mol Biol 22: 256-264, 2015.

30. Chen N, Zhao G, Yan X, Lv Z, Yin H, Zhang S, Song W, Li X, Li L, Du Z, et al: A novel FLI1 exonic circular RNA promotes metastasis in breast cancer by coordinately regulating TET1 and DNMT1. Genome Biol 19: 218, 2018.

31. Chen CY and Sarnow P: Initiation of protein synthesis by the eukaryotic translational apparatus on circular RNAs. Science 268: 415-417, 1995.

32. Legnini I, Di Timoteo G, Rossi F, Morlando M, Briganti F, Sthandier O, Fatica A, Santini T, Andronache A, Wade M, et al: Circ-ZNF609 is a circular RNA that can be translated and functions in myogenesis. Molecular Cell 66: 22-37.e9, 2017. 
33. Yang Y, Gao X, Zhang M, Yan S, Sun C, Xiao F, Huang N, Yang X, Zhao K, Zhou H, et al: Novel role of FBXW7 circular RNA in repressing glioma tumorigenesis. J Natl Cancer Inst 110: 304-315, 2018

34. Zhang M, Huang N, Yang X, Luo J, Yan S, Xiao F, Chen W, Gao X, Zhao K, Zhou H, et al: A novel protein encoded by the circular form of the SHPRH gene suppresses glioma tumorigenesis. Oncogene 37: 1805-1814, 2018.

35. Torre LA, Siegel RL, Ward EM and Jemal A: Global cancer incidence and mortality rates and trends-an update. Cancer Epidemiol Biomarkers Prev 25: 16-27, 2016.

36. Siegel RL, Miller KD and Jemal A: Cancer statistics, 2019. CA Cancer J Clin 69: 7-34, 2019.

37. Zhang S and Batur P: Human papillomavirus in 2019: An update on cervical cancer prevention and screening guidelines. Cleve Clin J Med 86: 173-178, 2019.

38. Yee GP, de Souza P and Khachigian LM: Current and potential treatments for cervical cancer. Curr Cancer Drug Targets 13 205-220, 2013

39. Bray F, Ferlay J, Soerjomataram I, Siegel RL, Torre LA and Jemal A: Global cancer statistics 2018: GLOBOCAN estimates of incidence and mortality worldwide for 36 cancers in 185 countries. CA Cancer J Clin 68: 394-424, 2018.

40. Mezei AK, Armstrong HL, Pedersen HN, Campos NG, Mitchell SM, Sekikubo M, Byamugisha JK, Kim JJ, Bryan S and Ogilvie GS: Cost-effectiveness of cervical cancer screening methods in low- and middle-income countries: A systematic review. Int J Cancer 141: 437-446, 2017.

41. Salzman J, Chen RE, Olsen MN, Wang PL and Brown PO Cell-type specific features of circular RNA expression. PLoS Genet 9: e1003777, 2013.

42. Han YN, Xia SQ, Zhang YY, Zheng JH and Li W: Circular RNAs: A novel type of biomarker and genetic tools in cancer. Oncotarget 8: 64551-64563, 2017.

43. Zheng SR, Zhang HR, Zhang ZF, Lai SY, Huang LJ, Liu J, Bai X, Ding K and Zhou JY: Human papillomavirus $16 \mathrm{E} 7$ oncoprotein alters the expression profiles of circular RNAs in Caski cells. J Cancer 9: 3755-3764, 2018.

44. Gao YL, Zhang MY, Xu B, Han LJ, Lan SF, Chen J, Dong YJ and Cao LL: Circular RNA expression profiles reveal that hsa_circ_0018289 is up-regulated in cervical cancer and promotes the tumorigenesis. Oncotarget 8: 86625-86633, 2017.

45. Jiao J, Zhang T, Jiao X, Huang T, Zhao L, Ma D and Cui B hsa_circ_0000745 promotes cervical cancer by increasing cell proliferation, migration, and invasion. J Cell Physiol 235 . 1287-1295, 2020.

46. Wang Y, Wang L, Wang W and Guo X: Overexpression of circular RNA hsa_circ_0001038 promotes cervical cancer cell progression by acting as a ceRNA for miR-337-3p to regulate cyclin-M3 and metastasis-associated in colon cancer 1 expression. Gene 733: 144273, 2019.

47. Chen RX, Liu HL, Yang LL, Kang FH, Xin LP, Huang LR, Guo QF and Wang YL: Circular RNA circRNA_0000285 promotes cervical cancer development by regulating FUS. Eur Rev Med Pharmacol Sci 23: 8771-8778, 2019.

48. Hu C, Wang Y, Li A, Zhang J, Xue F and Zhu L: Overexpressed circ 0067934 acts as an oncogene to facilitate cervical cancer progression via the miR-545/EIF3C axis. J Cell Physiol 234: 9225-9232, 2019.

49. Ding L and Zhang H: Circ-ATP8A2 promotes cell proliferation and invasion as a ceRNA to target EGFR by sponging miR-433 in cervical cancer. Gene 705: 103-108, 2019.

50. Ma H, Tian T, Liu X, Xia M, Chen C, Mai L, Xie S and Yu L: Upregulated circ_0005576 facilitates cervical cancer progression via the miR-153/KIF20A axis. Biomed Pharmacother 118: 109311, 2019

51. Jiao J, Jiao X, Liu Q, Qu W, Ma D, Zhang Y and Cui B: The Regulatory Role of circRNA_101308 in cervical cancer and the prediction of its mechanism. Cancer Manag Res 12: 4807-4815, 2020.

52. Ji F, Du R, Chen T, Zhang M, Zhu Y, Luo X and Ding Y: Circular RNA circSLC26A4 accelerates cervical cancer progression via miR-1287-5p/HOXA7 axis. Mol Ther Nucleic Acids 19: 413-420, 2020.

53. He J, Lv X and Zeng Z: A potential disease monitoring and prognostic biomarker in cervical cancer patients: The clinical application of circular RNA_0018289. J Clin Lab Anal 34: e23340, 2020.
54. Zhang J, Zhao X, Zhang J, Zheng $X$ and Li F: Circular RNA hsa_circ_0023404 exerts an oncogenic role in cervical cancer through regulating miR-136/TFCP2/YAP pathway. Biochem Biophys Res Commun 501: 428-433, 2018.

55. Tang Q, Chen Z, Zhao L and Xu H: Circular RNA hsa circ_0000515 acts as a miR-326 sponge to promote cervical cancer progression through up-regulation of ELK1. Aging (Albany NY) 11: 9982-9999, 2019.

56. Liu H, Wu Y, Wang S, Jiang J, Zhang C, Jiang Y, Wang X, Hong $\mathrm{L}$ and Huang $\mathrm{H}$ : Circ-SMARCA5 suppresses progression of multiple myeloma by targeting miR-767-5p. BMC Cancer 19: 937, 2019.

57. Dong C, Fan B, Ren Z, Liu B and Wang Y: CircSMARCA5 facilitates the progression of prostate cancer through miR-432/PDCD10 axis. Cancer Biother Radiopharm, 2020 (Online ahead of print)

58. Tian JDC and Liang L: Involvement of circular RNA SMARCA5/microRNA-620 axis in the regulation of cervical cancer cell proliferation, invasion and migration. Eur Rev Med Pharmacol Sci 22: 8589-8598, 2018.

59. Yang C, Yuan W, Yang X, Li P, Wang J, Han J, Tao J, Li P, Yang H, Lv Q and Zhang W: Circular RNA circ-ITCH inhibits bladder cancer progression by sponging miR-17/miR-224 and regulating p21, PTEN expression. Mol Cancer 17: 19, 2018.

60. Wang ST, Liu LB, Li XM, Wang YF, Xie PJ, Li Q, Wang R, Wei Q, Kang YH, Meng R and Feng XH: Circ-ITCH regulates triple-negative breast cancer progression through the Wnt/ $\beta$-catenin pathway. Neoplasma 66: 232-239, 2019.

61. Li J, Guo R, Liu Q, Sun J and Wang H: Circular RNA Circ-ITCH inhibits the malignant behaviors of cervical cancer by microRNA-93-5p/FOXK2 Axis. Reprod Sci 27: 860-868, 2020.

62. Cai H, Zhang P, Xu M, Yan L, Liu N and Wu X: Circular RNA hsa_circ_0000263 participates in cervical cancer development by regulating target gene of miR-150-5p. J Cell Physiol 234: 11391-11400, 2019.

63. Torre LA, Trabert B, DeSantis CE, Miller KD, Samimi G, Runowicz CD, Gaudet MM, Jemal A and Siegel RL: Ovarian cancer statistics, 2018. CA Cancer J Clin 68: 284-296, 2018.

64. Hackshaw A, Gershenson D and Ledermann J: Mucinous Ovarian Carcinoma. N Engl J Med 381: e3, 2019.

65. Wang L, Wang Q, Xu Y and Han L: Advances in the treatment of ovarian cancer using PARP inhibitors and the underlying mechanism of resistance. Current Drug Targets 21: 167-178, 2020.

66. Hansen JM, Coleman RL and Sood AK: Targeting the tumour microenvironment in ovarian cancer. Eur J Cancer 56: 131-143, 2016.

67. Teng F, Xu J, Zhang M, Liu S, Gu Y, Zhang M, Wang X, Ni J, Qian B, Shen R and Jia X: Comprehensive circular RNA expression profiles and the tumor-suppressive function of circHIPK3 in ovarian cancer. Int J Biochem Cell Biol 112: 8-17, 2019.

68. Ning L, Long B, Zhang W, Yu M, Wang S, Cao D, Yang J, Shen K, Huang Y and Lang J: Circular RNA profiling reveals circEXOC6B and circN4BP2L2 as novel prognostic biomarkers in epithelial ovarian cancer. Int J Oncol 53: 2637-2646, 2018.

69. Gao Y, Zhang C, Liu Y and Wang M: Circular RNA profiling reveals circRNA1656 as a novel biomarker in high grade serous ovarian cancer. Biosci Trends 13: 204-211, 2019.

70. Fan CM, Wang JP, Tang YY, Zhao J, He SY, Xiong F, Guo C, Xiang B, Zhou M, Li XL, et al: circMAN1A2 could serve as a novel serum biomarker for malignant tumors. Cancer Sci 110: 2180-2188, 2019.

71. Pei $\mathrm{C}$, Wang H, Shi $\mathrm{C}$, Zhang $\mathrm{C}$ and Wang M: CircRNA hsa_circ_0013958 may contribute to the development of ovarian cancer by affecting epithelial-mesenchymal transition and apoptotic signaling pathways. J Clin Lab Anal 34: e23292, 2020.

72. Zou T, Wang PL, Gao Y and Liang WT: Circular RNA_LARP4 is lower expressed and serves as a potential biomarker of ovarian cancer prognosis. Eur Rev Med Pharmacol Sci 22: 7178-7182, 2018.

73. Zhao Y, Hu Y, Shen Q, Chen Q, Zhu XJ, Jiang SS and Zhang Q: CircRNA_MYLK promotes malignant progression of ovarian cancer through regulating microRNA-652. Eur Rev Med Pharmacol Sci 24: 5281-5291, 2020.

74. Zhang M, Xia B, Xu Y, Zhang Y, Xu J and Lou G: Circular RNA (hsa_circ 0051240) promotes cell proliferation, migration and invasion in ovarian cancer through miR-637/KLK4 axis. Artif Cells Nanomed Biotechnol 47: 1224-1233, 2019. 
75. Chen Y, Ye X, Xia X and Lin X: Circular RNA ABCB10 correlates with advanced clinicopathological features and unfavorable survival, and promotes cell proliferation while reduces cell apoptosis in epithelial ovarian cancer. J Nurs Scholarsh 26: 151-161, 2019.

76. Zhao Y, Qin XP, Lang YP, Kou D and Shao ZW: Circular RNA circ-SMAD7 promoted ovarian cancer cell proliferation and metastasis by suppressing KLF6. Eur Rev Med Pharmacol Sci 23: 5603-5610, 2019.

77. Bao L, Zhong J and Pang L: Upregulation of Circular RNA VPS13C-has-circ-001567 promotes ovarian cancer cell proliferation and invasion. Cancer Biother Radiopharm 34: 110-118, 2019.

78. Chen H, Mao M, Jiang J, Zhu D and Li P: Circular RNA CDR1as acts as a sponge of miR-135b-5p to suppress ovarian cancer progression. Onco Targets Ther 12: 3869-3879, 2019.

79. Zhang N, Jin $\mathrm{Y}, \mathrm{Hu} \mathrm{Q}$, Cheng $\mathrm{S}$, Wang $\mathrm{C}$, Yang $\mathrm{Z}$ and Wang $\mathrm{Y}$ Circular RNA hsa_circ_0078607 suppresses ovarian cancer progression by regulating miR-518a-5p/Fas signaling pathway. J Ovarian Res 13: 64, 2020.

80. Li X, Lin S, Mo Z, Jiang J, Tang $\mathrm{H}$, Wu $\mathrm{C}$ and Song $\mathrm{J}$ : CircRNA_100395 inhibits cell proliferation and metastasis in ovarian cancer via regulating miR-1228/p53/epithelial-mesenchymal transition (EMT) axis. J Cancer 11: 599-609, 2020.

81. Wang W, Wang J, Zhang X and Liu G: Serum circSETDB1 is a promising biomarker for predicting response to platinum-taxane-combined chemotherapy and relapse in high-grade serous ovarian cancer. Onco Targets Ther 12 : 7451-7457, 2019

82. Zhao Z, Ji M, Wang Q, He N and Li Y: Circular RNA Cdrlas upregulates SCAI to suppress cisplatin resistance in ovarian cancer via miR-1270 suppression. Mol Ther Nucleic Acids 18 24-33, 2019.

83. Lortet-Tieulent J, Ferlay J, Bray F and Jemal A: International patterns and trends in endometrial cancer incidence, 1978-2013. J Natl Cancer Inst 110: 354-361, 2018.

84. McAlpine JN, Temkin SM and Mackay HJ: Endometrial cancer: Not your grandmother's cancer. Cancer 122: 2787-2798, 2016.

85. Braun MM, Overbeek-Wager EA and Grumbo RJ: Diagnosis and management of endometrial cancer. Am Fam Physician 93: 468-474, 2016.

86. Moore K and Brewer MA: Endometrial cancer: Is this a new disease? Am Soc Clin Oncol Educ Book 37: 435-442, 2017.
87. Urick ME and Bell DW: Clinical actionability of molecular targets in endometrial cancer. Nat Rev Cancer 19: 510-521, 2019.

88. Bokhman JV: Two pathogenetic types of endometrial carcinoma. Gynecol Oncol 15: 10-17, 1983.

89. Suarez AA, Felix AS and Cohn DE: Bokhman Redux: Endometrial cancer 'types' in the 21st century. Gynecol Oncol 144: 243-249, 2017.

90. Cancer Genome Atlas Research Network, Kandoth C, Schultz N, Cherniack AD, Akbani R, Liu Y, Shen H, Robertson AG, Pashtan I, Shen R, et al: Integrated genomic characterization of endometrial carcinoma. Nature 497: 67-73, 2013.

91. Colombo N, Creutzberg C, Amant F, Bosse T, González-Martín A, Ledermann J, Marth C, Nout R, Querleu D, Mirza MR, et al: ESMO-ESGO-ESTRO Consensus Conference on Endometrial Cancer: diagnosis, treatment and follow-up. Ann Oncol 27: $16-41,2016$.

92. Ye F, Tang QL, Ma F, Cai L, Chen M, Ran XX, Wang XY and Jiang XF: Analysis of the circular RNA transcriptome in the grade 3 endometrial cancer. Cancer Manag Res 11: 6215-6227, 2019.

93. Xu H, Gong Z, Shen Y, Fang Y and Zhong S: Circular RNA expression in extracellular vesicles isolated from serum of patients with endometrial cancer. Epigenomics 10: 187-197, 2018.

94. Chen BJ, Byrne FL, Takenaka K, Modesitt SC, Olzomer EM, Mills JD, Farrell R, Hoehn KL and Janitz M: Analysis of the circular RNA transcriptome in endometrial cancer. Oncotarget 9: 5786-5796, 2018

95. Wang H, Douglas W, Lia M, Edelmann W, Kucherlapati R, Podsypanina K, Parsons R and Ellenson LH: DNA mismatch repair deficiency accelerates endometrial tumorigenesis in Pten heterozygous mice. Am J Pathol 160: 1481-1486, 2002.

96. Stambolic V, Tsao MS, Macpherson D, Suzuki A, Chapman WB and Mak TW: High incidence of breast and endometrial neoplasia resembling human Cowden syndrome in pten $+/-$ mice. Cancer Res 60: 3605-3611, 2000.

97. Liu Y, Chen S, Zong ZH, Guan X and Zhao Y: CircRNA WHSC1 targets the miR-646/NPM1 pathway to promote the development of endometrial cancer. J Cell Mol Med 24: 6898-6907, 2020. International (CC BY-NC-ND 4.0) License. 